\title{
A CONTRIBUIÇÃO DAS FERRAMENTAS VISUAIS PARA O PROCESSO DE DESENVOLVIMENTO DE PRODUTOS, UMA REVISÃO SISTEMÁTICA DA LITERATURA
}

[ Autor 1 ] Ana Cláudia Costa de Araújo (anaclaudiacostaaraujo@gmail.com), Departamento de Engenharia de Produção, Universidade Federal do Rio Grande do Norte.

[ Autor 2 ] Marcela Silva Cavalcanti Rosa (marcela.cavalcantir@gmail.com), Departamento de Engenharia de Produção, Universidade Federal do Rio Grande do Norte.

[ Autor 3] Técia de Lima Silva (anaclaudiacostaaraujo@gmail.com), Departamento de Engenharia de Produção, Universidade Federal do Rio Grande do Norte.

[ Autor 4 ] George André Souza Santiago (geosantiago@yahoo.com.br), Departamento de Engenharia de Produção, Universidade Federal do Rio Grande do Norte.

[ Autor 5 ] Mario Orestes Aguirre González (mario@ct.ufrn.br), Departamento de Engenharia de Produção, Universidade Federal do Rio Grande do Norte.

\section{RESUMO}

A sociedade atual vive diante de um grande fluxo de informações e, por consequência, há uma necessidade de assimilação de conteúdo cada vez mais rápida, sem comprometer a eficácia da informação transmitida. Portanto, é preciso comunicar-se de forma simples e intuitiva o que é possível por meio da configuração visual. Dessa forma, as ferramentas visuais são aliadas eficientes para o desenvolvimento de produtos inovadores. O processo de design do produto, especialmente a fase de geração de ideias, fornece uma oportunidade para a aplicação do pensamento criativo à engenharia. Nesse contexto, este estudo tem o intuito de mapear ferramentas visuais que auxiliam no processo de desenvolvimento do produto. Para tanto, foi realizada uma revisão bibliográfica sistemática nas plataformas Portal Capes, Science Direct e Google Acadêmico, mediante a análise de 60 artigos sobre comunicação visual e visualização da informação, coletados por meio de um método de cinco etapas. Como resultado, os artigos explorados demonstraram que as ferramentas visuais podem auxiliar no processo de criatividade e inovação no design do produto. Dentre as técnicas mapeadas, pode-se perceber sua aplicabilidade visando gerar não só ideias, mas também facilitar processos, assimilação de informações, estimulação do intercâmbio de conceitos entre equipes, contribuição para solução de problemas e propostas de soluções de design. Apresentou-se as ferramentas visuais vinculadas ao estímulo da criatividade: mood board, representação pictórica, imagery, sketches, esboços heurísticos, diagrama conceitual e de conexão. O agrupamento das funções destas ferramentas contribui para a visualização da ideia de forma mais detalhada, precisa e com participação coletiva dos demais envolvidos no processo.

Palavras-chave: Revisão Bibliográfica Sistemática, Ferramentas Visuais, Desenvolvimento de Produtos.

Área: Ferramentas e métodos de desenvolvimento de produtos e serviços. 


\section{INTRODUÇÃO}

A visualização da informação tem sua base vinculada à teoria da comunicação visual cujo conceito surgiu desde as primícias da linguagem, onde o código verbal era inexistente e a única forma de comunicação era mediante pinturas rupestres. Mensagens com conteúdo visual, em diferentes formas, tornaram-se cada vez mais importantes com o passar do tempo (PETTERSSON, 1996). A evolução da linguagem trouxe a configuração do código verbal, contudo a dinamicidade das relações interpessoais e organizacionais levaram tal linguagem de volta ao patamar visual. Estrada e Davis (2014) apontam a cultura visual como um importante fator na identidade cultural da sociedade do século XXI, no qual as imagens tornaram-se uma ferramenta fundamental com a qual se comunica ciência.

Atualmente a sociedade vive diante de um grande fluxo de informações. Em contrapartida, há a necessidade de assimilar os conteúdos de forma cada vez mais rápida, mas sem comprometer a eficácia da informação transmitida. Portanto, deve-se buscar comunicar-se com configuração visual para tornar a mensagem simples, clara e intuitiva. Para Lurie e Mason (2007) as representações visuais da mensagem podem ampliar as capacidades de resolução de problemas permitindo o processamento da informação visual de maneira mais rápida e eficaz. Suas aplicações podem melhorar a capacidade do indivíduo de processar a informação (TEGARDEN, 1999).

Sob esta ótica, Adiloglu (2011) afirma que a informação visual eficaz habita na criatividade, além de pertencer a um campo interdisciplinar. Logo, as ferramentas visuais são fortes aliadas para o desenvolvimento de produtos inovadores. O processo de concepção do produto, em especial a fase da geração da ideia, fornece uma oportunidade para aplicação do pensamento criativo à engenharia (AMABILE, 1983). Representar a mensagem de forma visual, mediante ferramentas adequadas, é fundamental para a elaboração e inovação de produtos. Assim, a criatividade e as ferramentas visuais podem possibilitar a geração de ideias criativas.

Diante disso, este trabalho tem o objetivo de mapear ferramentas visuais úteis no processo de desenvolvimento do produto. Para tal finalidade, foi realizada uma revisão bibliográfica sistemática a fim de catalogar essas ferramentas discutidas na literatura.

O artigo estrutura-se da seguinte forma: na primeira seção apresenta-se a introdução, seguida pelo detalhamento do método de pesquisa utilizado. A terceira e quarta seções referem-se à fundamentação teórica sobre as temáticas: visualização da informação, criatividade e desenvolvimento do produto. $\mathrm{Na}$ seção cinco denotam-se os resultados, mediante a apresentação da tabela composta pelas ferramentas visuais mapeadas. Por fim, a conclusão é apresentada na sexta seção.

\section{MÉTODO}

Para fornecer suporte à fundamentação teórica deste estudo, a pesquisa e seleção dos artigos foi realizada de forma sistemática, organizada e subdividida em cinco etapas. Inicialmente foram delineados o problema de pesquisa e os objetivos para a definição das palavras-chaves mais apropriadas ao estudo. Uma vez que esta fase foi executada, a segunda etapa corresponde à seleção das bases de dados em que a pesquisa será realizada. Para isso, utilizouse as bases científicas: Portal Capes, Science Direct e Google Acadêmico. Assim sendo, inseriu-se as seguintes palavras-chaves: "visual communication", "visual tools"; "visual literacy"; "visual literacy" + "visual communication"; "visual practices" + "communication". Os filtros adotados para a seleção dos artigos abarcam o alinhamento com o problema de pesquisa e o ínterim de publicação, que deve estar inserido no período estipulado entre 2005 e 2016 . Como resultado da coleta dos artigos, atingiu-se 33 publicações a partir da leitura preliminar (título, resumo e introdução). 
A terceira etapa da revisão bibliográfica sistemática (RBS) contemplou uma leitura completa dos 33 artigos coletados, cujo conteúdo deveria abranger comunicação visual, ferramentas visuais e visualização da informação. No entanto apenas 23 destes artigos se mostraram alinhados ao problema de pesquisa. Na quarta etapa estes artigos tiveram as suas referências verificadas e assim, atingiu-se um novo montante de publicações condizentes com a problemática de estudo, totalizando 63 artigos cujo conteúdo foi analisado. Por fim, realizouse o mapeamento das ferramentas visuais existentes na literatura.

\section{VISUALIZAÇÃO DA INFORMAÇÃO E FERRAMENTAS VISUAIS}

\subsection{Ramificações da Visualização da Informação}

O processo de visualizar a informação se apresenta como solução para o excesso de conhecimentos e dados em que o mundo está imerso. As aplicações dessas técnicas auxiliam o indivíduo a processar a informação visual de forma mais concisa e eficaz. As técnicas de visualização pertencem à um amplo campo de aplicação com possibilidades nas áreas de gestão de negócios, capacitação de pessoas, elaboração de estratégia, dentre outras.

Segundo Tegarden (1999) a visualização tem o propósito de permitir a exploração do sistema visual humano a fim de coletar informações oriundas de dados. Logo, a abordagem também pode fornecer um panorama mais claro quando se refere a dados complexos. Em suma, o estudo apresenta pesquisas sobre cognição humana, na qual a visualização é exibida como ferramenta, cuja propriedade é ampliar a capacidade do indivíduo em tarefas de resolução de problemas.

No que refere-se à cognição humana, Smiciklas (2012) relata que metade do cérebro humano está envolvido de forma direta ou indireta no processamento da mensagem visual, onde $30 \%$ do tecido cerebral é composto por neurônios envolvidos em atividades visuais. Atualmente, a sociedade vive uma época de cultura visual (AVGERINOU; ERICSON, 1997) e em essência o aumento gradativo do fluxo de informações, o que torna cada vez mais substancial a visualização da informação e a evolução visual do indivíduo.

O uso de ferramentas de visualização vai permitir que soluções de negócios sejam potencializadas de acordo com as necessidades propostas. Todavia, Zhang (2012) afirma que a visualização pode ser definida como um tipo de linguagem visual utilizada para representar de maneira estática ou dinâmica conceitos ou conjunto de dados em uma ou mais dimensões.

A visualização também pode ser retratada como a representação gráfica ou mapeamento de informações de uma forma espacial, além de ser um termo utilizado com frequência por estudiosos da comunicação visual (KERBANCH; EPPLER; BRESCIANI, 2015). Diante disso, Eppler e Bresciani (2015) apresentam estudos que englobam as limitações da visualização e a definem como uma exibição gráfica de informação e conhecimento. No que tange a aplicação dessas ferramentas, a pesquisa realizada por Dahl e Gorn (2001) aborda a visualização como ferramenta facilitadora da concepção de projetos durante o processo de design. Para estes pesquisadores a visualização permite a geração, interpretação e manipulação da informação através da representação espacial.

De acordo com Emanuel e Lipton (2013) um grande contingente de pessoas tem tendência a pensar mais em palavras do que em imagens, contudo, o uso da visualização no pensamento do indivíduo vem crescendo e se moldando às suas necessidades. Além de auxiliar na visualização de dados, o uso de abordagens visuais facilitará a compreensão e interpretação das informações colocadas. Zhang (2012) ratifica esse entendimento por intermédio do seu estudo sobre a importância da visualização para promover uma comunicação rápida e eficaz 
no campo da gestão de negócios. Sua pesquisa ainda aborda exemplos de aplicações da visualização em áreas da gestão, tais quais: finanças, organização, gestão e a representação visual de conceitos gerenciais.

Em suma, a prática de visualizar a informação configura a formulação de uma imagem mental com englobamento de dados diversos, que ao serem representados de forma espacial, permitem que o indivíduo decodifique a mensagem de forma mais concisa e eficaz. A representação gráfica de dados proporciona a construção de um formato visual gráfico, orientado por elementos visuais, alinhados ao sistema de cognição visual humano. Desta forma, a abordagem visual torna-se apta para aplicação na área de resolução de problemas, extraindo dos dados não só informação bruta, mas também interpretação e processamento da mensagem que se deseja transmitir, mediante representação espacial.

A Tabela 1 apresenta as definições de diferentes autores, encontradas na literatura, sobre visualização da informação.

Tabela 1 - Evolução dos conceitos de visualização

\begin{tabular}{|c|c|}
\hline Autor & Conceito \\
\hline $\begin{array}{c}\text { Dahl, Chattopadhyay e } \\
\text { Gorn (2001) }\end{array}$ & $\begin{array}{c}\text { A visualização permite a geração, interpretação e manipulação da informação } \\
\text { através da representação espacial. }\end{array}$ \\
\hline Platts e Tan (2004) & $\begin{array}{c}\text { A visualização transforma os dados brutos em uma forma acessível de } \\
\text { representação do conhecimento. }\end{array}$ \\
\hline Lurie e Mason (2007) & $\begin{array}{c}\text { Visualização envolve a seleção, a transformação, e apresentação de em uma } \\
\text { forma visual que facilita a exploração e compreensão. }\end{array}$ \\
\hline Lurie e Mason (2007) & $\begin{array}{c}\text { Visualizar refere-se à um processo que extrai informação significativa a partir } \\
\text { dos dados, e constrói uma representação visual da informação. }\end{array}$ \\
\hline Eppler e Bresciani (2013) & $\begin{array}{c}\text { Visualização aparece como uma estratégia óbvia para lidar com os riscos de } \\
\text { sobrecarga de informação. }\end{array}$ \\
\hline Cybulski et al. (2013) & $\begin{array}{c}\text { A visualização também é considerada um elemento-chave na resolução de } \\
\text { problemas. }\end{array}$ \\
\hline
\end{tabular}

\subsection{Ferramentas visuais}

A prática de visualizar a informação configura a formulação de uma imagem mental que engloba dados diversos, que ao serem representados de forma espacial, permitem que o indivíduo decodifique a mensagem de forma mais concisa e eficaz. A representação gráfica de dados, ou seja, a visualização da informação proporciona a construção de um formato visual gráfico, orientado por elementos visuais alinhados ao sistema de cognição visual humano. Desta forma, a abordagem visual torna-se apta para aplicação na área de resolução de problemas, extraindo dos dados não só informação bruta, mas também interpretação e processamento da mensagem que se deseja transmitir, mediante representação espacial.

Cybulski et al. (2013) destacam a visualização como uma ferramenta importante na resolução de problemas e referem-se à visualização de dados não só como abordagem voltada à compreensão de dados e novos conhecimentos, mas também como ferramenta de apresentação cujas aplicações são: ilustrar, explicar, e comunicar resultados. Ao apresentar 
esses dados em um formato visual, a visualização auxiliará também no processo de tomada de decisão, ao atuar como ferramenta de gestão.

Antigamente a visualização era utilizada na área de gestão apenas por meio de gráficos estatísticos, o que apesar de permitir ao gestor a visualização dos dados, não se adequa, devido ao seu formato engessado, à sobrecarga de informação na qual as empresas periodicamente estão inseridas. Como alternativa aos gráficos, Eppler e Bresciani (2013) apontam como solução as ferramentas de visualização qualitativas, tais quais metáforas visuais, diagramas, dentre outros que têm o intuito de facilitar tarefas durante a etapa de gerar ideias para aplicá-las na tomada de decisão, como também no planejamento e aprendizagem. Para estes autores a visualização não deve ser apenas uma ferramenta de exibição e compilação de dados, e sim uma abordagem colaborativa que auxiliará na gestão e também no processo criativo das tomadas de decisões.

Eppler (2006) entende que a principal vantagem de uma sumária representação visual de um conceito é que, desta forma, se pode obter uma visão sistemática de maneira breve não apenas descrevendo o conteúdo, mas também podendo analisá-lo quanto à sua natureza epistêmica. Neste viés, Eppler e Burkhard (2007) reiteram que ferramentas de visualização são baseadas em regras e representações gráficas, que descrevem as informações de forma mais adequada a aquisição de conhecimentos a fim de proporcionar uma compreensão elaborada ou novas experiências de comunicação.

$\mathrm{Na}$ literatura pode-se encontrar o estudo de variadas ferramentas/abordagens/práticas visuais em caráter multidisciplinar, devido sua utilização não estar restrita apenas às áreas de design e comunicação, podendo-se aplicar também aos campos de gestão e engenharia. Estas ferramentas não apresentam uma estrutura padrão, contudo, para representarem determinados conceitos os elementos visuais auxiliam no ofício de visualizar a informação desejada da melhor maneira possível.

\section{CRIATIVIDADE E DESENVOLVIMENTO DE PRODUTO}

A criatividade tem seu habitat na novidade. É a partir do diferente que ideias novas são geradas e aplicadas em ambientes organizacionais. Amabile (1996) define este construto como a semente de toda a inovação. No que concerne o indivíduo criativo, Sternberg (1988) coloca que as pessoas criativas têm uma combinação especial de atributos de personalidade, inteligência e estilo cognitivo. Diante de tais características, pessoas que apresentam perfil criativo geram respostas (AMABILE, 1996).

Sok e O'cass (2015) afirmam que a presença da criatividade é essencial no decorrer do processo de um produto inovador e de alta qualidade. Neste viés, Sawyer (2012) apresenta a criatividade como as ideias ou produtos gerados por indivíduos, enquanto a inovação é a execução bem-sucedida de um novo produto ou serviço realizado por uma empresa. Assim, é importante estudar esse construto junto ao processo de inovação, no intuito de promover melhorias no produto que será entregue ao mercado.

Para Sarooghi et al. (2015) o processo de inovação consiste em duas atividades principais: criatividade e inovação. A criatividade refere-se à geração de novas e úteis ideias, enquanto a inovação implementa as ideias geradas em novos produtos, ou processos. Sob esta ótica percebe-se a importância de alinhar estes dois construtos ao processo de desenvolvimento de produtos, visto que diante de uma sociedade consumidora cada vez mais exigente é necessário desenvolver produtos que atendam às expectativas do público-alvo desejado, proporcionando ao consumidor não só um item funcional no quesito qualidade, mas também com valor, conferindo vantagem competitiva a empresa. 
Neste contexto, salienta-se que a criatividade de uma ideia não depende apenas do conteúdo, mas da maneira com que essa ideia é desenvolvida, apresentada e interpretada (BILTON, 2007). Denomina-se desenvolvimento de produto a área que cuida de todos os estudos e pesquisas sobre criação, adaptação, melhorias e aprimoramento dos produtos produzidos pela empresa (CHIAVENATO, 2005). Assim, é importante estruturar o ambiente organizacional de forma que fomente a criatividade durante a elaboração/melhoria de um produto.

As ideias criativas são as responsáveis por conduzir o processo de inovação, além de estimular e produzir estas dentro do ambiente organizacional. Isto posto, o potencial criativo é inerente a todo indivíduo, contudo para que seja potencializado ele deve ser estimulado de forma correta e auxiliado pelas ferramentas adequadas, para que a geração de ideias, durante a etapa de desenvolvimento do produto, ocorra com eficácia e alcance os objetivos desejados tanto em índices de inovação quanto de criatividade.

\section{RESULTADOS}

A Tabela apresentada abaixo identifica as principais ferramentas/práticas/métodos visuais identificados neste estudo. As ferramentas mapeadas fazem parte de diferentes áreas de estudo com aplicabilidade multidisciplinar, tais como design, comunicação, gestão e engenharias.

Tabela 2 - Mapeamento das ferramentas visuais

\begin{tabular}{|c|c|c|}
\hline $\begin{array}{l}\text { Técnica / Prática } \\
\text { / Ferramenta } \\
\text { Visual }\end{array}$ & Descrição & Fonte \\
\hline Imagem (Imagery) & $\begin{array}{c}\text { Ferramenta poderosa, na publicidade ou vida real, para a } \\
\text { implantação de ideias ou sugestões, induzindo emoções, ou } \\
\text { virando uma ideia abstrata em benefício do produto real e credível. }\end{array}$ & $\begin{array}{l}\text { Branthwaite } \\
\qquad(2002) ; \\
\text { Burkhard (2005) }\end{array}$ \\
\hline Papel Paisagem & $\begin{array}{c}\text { Documento auxiliar de design, que permite aos designers e } \\
\text { interessados a gravar, explorar e avaliar vários designs } \\
\text { alternativos. }\end{array}$ & Brath (2003) \\
\hline $\begin{array}{c}\text { Perfil de } \\
\text { desempenho }\end{array}$ & $\begin{array}{c}\text { Fornece comparação visual clara, permitindo que os gerentes } \\
\text { avaliem o alinhamento que existe, para testar a correlação entre as } \\
\text { exigências do mercado e o desempenho alcançado. }\end{array}$ & $\begin{array}{l}\text { Platts e Tan } \\
\qquad(2004)\end{array}$ \\
\hline $\begin{array}{l}\text { TAPS (Tool for } \\
\text { Action Plan } \\
\text { Selection) }\end{array}$ & $\begin{array}{l}\text { Ferramenta de software para ajudar os gerentes a visualizar as } \\
\text { relações de causa-efeito entre as variáveis e objetivos. Assim, } \\
\text { permite aos gestores mapear as variáveis, ver como elas se } \\
\text { encaixam e como influenciam um ao outro, fazendo esta nova } \\
\text { geração e compreensão de ideias. }\end{array}$ & \\
\hline $\begin{array}{l}\text { Protocolos visuais } \\
\text { OnTrack }\end{array}$ & $\begin{array}{l}\text { Estrutura o intercâmbio de conhecimentos em fases divergentes e } \\
\text { convergentes. Proporciona, assim, uma visão geral do processo de } \\
\text { comunicação de conhecimentos. }\end{array}$ & Eppler (2004) \\
\hline $\begin{array}{l}\text { Aplicação de } \\
\text { parâmetros } \\
\text { Ruleris }\end{array}$ & $\begin{array}{l}\text { Visualiza a avaliação coletiva de critérios e avaliações de um } \\
\text { grupo de especialistas e tomadores de decisão. }\end{array}$ & \\
\hline
\end{tabular}




\begin{tabular}{|c|c|c|}
\hline Mapa de Sinergia & $\begin{array}{l}\text { Ajuda a identificar e discutir os objetivos de uma equipe e sua } \\
\text { interdependências em termos de sinergias e possíveis conflitos - } \\
\text { meta. Ao alinhar todos os objetivos da equipe, de acordo com o } \\
\text { seu horizonte de tempo e a importância, a discussão de parâmetros } \\
\text { de cada meta pode ser representada graficamente. }\end{array}$ & \\
\hline $\begin{array}{l}\text { Representação } \\
\text { Pictórica } \\
\text { (Expressão Visual) }\end{array}$ & $\begin{array}{c}\text { Técnica fundamental no processo originário de ideias para novos } \\
\text { produtos. A utilização destas formas de representação pictórica } \\
\text { tem sido considerada para se relacionar com criatividade e } \\
\text { inovação no design. }\end{array}$ & $\begin{array}{l}\text { Liu, Tang, } \\
\text { Frazer (2004) }\end{array}$ \\
\hline Mood Board & $\begin{array}{l}\text { Coleção de imagens que permite que o designer visualize ideias ou } \\
\text { emoções durante o processo de concepção do produto. Esta } \\
\text { ferramenta pode inspirar a aperfeiçoar suas soluções de design. }\end{array}$ & $\begin{array}{l}\text { Mc Donagh et } \\
\text { al. (2005) }\end{array}$ \\
\hline Sketches & $\begin{array}{l}\text { Representam a ideia principal e ajudam a rapidamente visualizar } \\
\text { uma ideia. }\end{array}$ & Burkhard (2005) \\
\hline Metáforas Visuais & Apoiam recall, o raciocínio e comunicação. & \\
\hline Texto Estruturado & $\begin{array}{l}\text { Formatação de texto, por exemplo, através do destaque de } \\
\text { palavras, formatação de parágrafos, usando diferentes cores, fontes } \\
\text { e tamanhos de fonte. Um segundo passo é a integração dos itens } \\
\text { textuais em estruturas sobrepostas visuais, tais como uma estrutura } \\
\text { de árvore ou uma tabela. }\end{array}$ & $\begin{array}{c}\text { Eppler e } \\
\text { Burkhard (2007) }\end{array}$ \\
\hline $\begin{array}{l}\text { Esboços } \\
\text { Heurísticos }\end{array}$ & $\begin{array}{l}\text { Desenhos que são usados para ajudar o processo de comunicação, } \\
\text { tornando o conhecimento em andamento explícito e discutível. Sua } \\
\text { função é representar a ideia principal e as principais características } \\
\text { de uma visão preliminar. }\end{array}$ & \\
\hline $\begin{array}{l}\text { Diagramas } \\
\text { Conceituais }\end{array}$ & $\begin{array}{l}\text { Representações esquemáticas de ideias abstratas com a ajuda de } \\
\text { formas padronizadas (tais como setas, círculos, pirâmides ou } \\
\text { matrizes). Eles são usados para estruturar informações e ilustrar as } \\
\text { relações. }\end{array}$ & \\
\hline Mapas Conceituais & $\begin{array}{l}\text { Uma forma de diagrama, que também faz uso de representações } \\
\text { esquemáticas para visualizar as relações entre conceitos. Um mapa } \\
\text { conceito geralmente consiste em dois elementos: um item e uma } \\
\text { relação entre dois itens. }\end{array}$ & $\begin{array}{l}\text { Eppler (2006); } \\
\text { Eppler e } \\
\text { Burkhard (2007) }\end{array}$ \\
\hline $\begin{array}{l}\text { Mapa de } \\
\text { Conhecimento }\end{array}$ & $\begin{array}{l}\text { Formatos gráficos que seguem convenções cartográficas para } \\
\text { referenciar o conhecimento relevante. }\end{array}$ & $\begin{array}{c}\text { Eppler e } \\
\text { Burkhard (2007); }\end{array}$ \\
\hline $\begin{array}{l}\text { Diagrama de } \\
\text { Conexão }\end{array}$ & $\begin{array}{l}\text { Apresenta os melhores resultados para estimular a criatividade, no } \\
\text { entanto, as estruturas de ligação são usadas para transmitir menos } \\
\text { informação. Usados para representar palavras, ideias, tarefas ou } \\
\text { outros itens em torno de uma palavra-chave ou ideia central. }\end{array}$ & $\begin{array}{l}\text { Rivera et al. } \\
\quad(2010)\end{array}$ \\
\hline Displays Visuais & $\begin{array}{l}\text { Ferramenta utilizada para resolver problemas de design, que } \\
\text { contribui para o reforço da resolução de problemas. }\end{array}$ & $\begin{array}{l}\text { Hoseini et al. } \\
\quad \text { (2013) }\end{array}$ \\
\hline
\end{tabular}




\begin{tabular}{|c|c|c|}
\hline $\begin{array}{l}\text { IVA (Análises } \\
\text { Visuais } \\
\text { Interativas) }\end{array}$ & $\begin{array}{c}\text { Abordagem visual para raciocínio analítico, que colocou em } \\
\text { movimento uma mudança de paradigma para a manipulação de } \\
\text { dados massivos, abertos e complexos. Combina percepção } \\
\text { humana, cognição e interação para processar grandes conjuntos de } \\
\text { dados. }\end{array}$ & $\begin{array}{c}\text { Cybulski et al } \\
\text { (2013) }\end{array}$ \\
\hline $\begin{array}{l}\text { VPM (visual ou } \\
\text { mapeamento de } \\
\text { projeto gráfico) }\end{array}$ & $\begin{array}{c}\text { Ferramenta que exibe cada projeto como um nó na rede e usa setas } \\
\text { para identificar relacionamentos ou interdependências entre os nós. } \\
\text { A criação de monitores VPM são auxiliados por softwares de } \\
\text { mapeamento de rede. }\end{array}$ & Killen (2013) \\
\hline $\begin{array}{c}\text { Análise de Rede } \\
\text { Social }\end{array}$ & $\begin{array}{c}\text { Uma das aplicações mais comuns de mapeamento de rede em que } \\
\text { as relações entre as pessoas ou organizações são apresentadas e } \\
\text { analisadas em uma forma. }\end{array}$ & \\
\hline
\end{tabular}

Intrínseco às ferramentas mapeadas neste estudo, pode-se perceber que algumas delas possuem aspectos que convergem no âmbito da geração de ideias, entretanto outras práticas mapeadas têm sua função principal voltada a visualização e alinhamento de conceitos para o desenvolvimento de estratégias no ambiente organizacional. Na configuração das ferramentas vinculadas a ideação, tais quais mood board, representação pictórica, imagery, sketches, esboços heurísticos, diagrama conceitual e de conexão, percebe-se a inserção de figuras compostas pela organização de elementos visuais básicos.

Para auxiliar no processo de desenvolvimento do produto, pode-se perceber que as ferramentas visuais, com aplicação na geração de ideias, têm funções que podem ser relevantes na construção da ideia de um novo produto. As aplicações dessas ferramentas proporcionam ao processo de ideação:

- Visualização da ideia de forma esquemática;

- Auxílio no processo de concepção de design do produto;

- Ilustração de relações entre as ideias geradas;

- Aperfeiçoamento de soluções de design;

- Implantação das ideias geradas no processo

O agrupamento destas funções acarretará uma ferramenta visual que viabiliza a visualização da ideia de forma compartilhada, em que ela será detalhada com mais profundidade e maior entendimento.

\section{CONCLUSÃO}

Ainda que a literatura contemple diversas ferramentas visuais de campos de pesquisa distintos, os estudos sobre visualização podem ser considerados algo novo, pois muitas dessas ferramentas surgiram com a evolução do indivíduo e a necessidade de otimizar processos, mediante a simplificação das informações.

Atualmente a sociedade demanda maior presença de informação visual no seu cotidiano, visto que as relações mercadológicas emergem com dinamicidade nos ambientes corporativos. Logo, torna-se essencial alinhar os construtos sobre criatividade e informação visual a favor do desenvolvimento de produtos cada vez melhores e inovadores para o mercado.

Os trabalhos explorados demonstraram que as ferramentas visuais podem auxiliar a proporcionar inovação e criatividade no processo de concepção do produto. Dentre as técnicas mapeadas, percebe-se suas aplicabilidades voltadas a gerar não só ideias, como também 
facilitar processos, assimilação de informações, estímulo do intercâmbio de conceitos entre equipes, contribuição para a resolução de problemas e propostas para soluções de design.

Mapear essas ferramentas permitiu analisar pesquisas sobre métodos visuais antigos e novos, conhecendo suas aplicações e funções que abrangem o processo de desenvolvimento de produto. Porém, devido sua flexibilidade essas ferramentas podem ser executadas em setores diferentes, visando melhorias através da utilização de práticas visuais.

No tocante à evolução deste estudo, é importante o entendimento dessas ferramentas quanto sua natureza epistêmica, visando os elementos visuais pelos quais ela é composta. Desta forma, em estudos futuros o intuito é entender a composição destas ferramentas visuais e, assim, construir uma nova ferramenta visual para fomentar inovação e criatividade no campo da engenharia.

\section{REFERÊNCIAS}

ADILOGLU, F. Visual communication: design studio education through working the process. Procedia - Social and Behavioral Sciences. v. 28, p. 982 - 991, 2011.

AMABILE, T. M. The social psychology of creativity: A componential conceptualization. Journal of personality and social psychology. V. 45, 1983.

AMABILE, T. M. Creativity in Context. Boulder, Colorado: Westview, 1996.

AVGERINOU, M.; ERICSON, J. A review of the concept of Visual Literacy. British Journal of Educational Technology. v. 28, p.280-291, 1997.

BILTON, C. Management and creativity: From creative industries to creative management. Malden, MA: Blackwell, 2007.

BRANTHWAITE, A. Investigating the power of imagery in marketing communication: evidence-based techniques. Qualitative Market Research: An International Journal. v. 5, p.164-171, 2002.

BRATH, R. Paper Landscapes: A Visualization Design Methodology. Visualization and Data Analysis. v. 5009, p.125-132, 2003.

BRESCIANI, S.; BLACKWELL, A.F.; EPPLER, M. J. A collaborative dimensions framework: Understanding the mediating role of conceptual visualizations in collaborative knowledge work. Proceedings of the Annual Hawaii International Conference on System Sciences. p. 1-10, 2008.

BRESCIANI, S.; EPPLER, M. J. The Pitfalls of Visual Representations: A Review and Classification of Common Errors Made While Designing and Interpreting Visualizations. SAGE Open. p.14, 2015.

BURKHARD, R. A. Strategy Visualization: A New Research Focus in Knowledge Visualization and a Case Study. Proceedings of I-KNOW'05. p. 1-8, 2005.

CYBULSKI, J. L.; KELLER, S.; NGUYEN L.; SAUNDAGE, D. Creative problem solving in digital space using visual analytics. Computers in Human Behavior. v. 42, p. 20-35, 2015.

DAHL, D.W.; CHATTOPADHYAY, A.; GORN, G. J. The importance of visualisation in concept design. Design Studies. v. 22, p.5-26, 2001.

EMANUEL, R.; LIPTON S. Visual Literacy and the Digital Native: Another Look. Journal of Visual Literacy. v. 32, p.7-26, 2013. 
EPPLER, M. J. A comparison between concept maps, mind maps, conceptual diagrams, and visual metaphors as complementary tools for knowledge construction and sharing. Information Visualization. v. 5, p.202-210, 2006.

EPPLER, M. J. Facilitating Knowledge Communication through Joint Interactive Visualization. Journal of Universal Computer Science. v. 10, pp.683-690, 2004.

EPPLER, M.J. BRESCIANI, S. Journal of Visual Languages and Computing Visualization in management: From communication to collaboration. A response to Zhang. Journal of Visual Language and Computing. v. 24, pp.146-149, 2013.

EPPLER, M. J.; BURKHARD, R. A. Visual representations in knowledge management: framework and cases. Journal of Knowledge Management. v. 11, p.112-122, 2007.

HONG LIU, M.; TANG, J.H.F. Supporting creative design in a visual evolutionary computing environment. Proceedings, DELTA 2004 - Second IEEE International Workshop on Electronic Design, Test and Applications, v. 35, pp.261-266, 2004.

HOSEINI, B.; MOZAFFAR, F.; AZIMI, M. Using visual displays to stimulate creativity: Implications for engineering design. International Journal of Advances in Engineering \& Technology. v. 5, p.198-206, 2013.

JÄNICKE, H.; WEIDNER, T.; CHUNG, D.; LARAMEE, R.; TOWNSEND, P; CHEN, M. Visual reconstructability as a quality metric for flow visualization. Computer Graphics Forum. v. 30, p.781-790, 2011.

KILLEN, C.P. Evaluation of project interdependency visualizations through decision scenario experimentation. International Journal of Project Management, v. 31(6), pp.804-816, 2013.

LURIE, N. H.; MASON, C. H. Visual Representation: Implications for Decision Making. Journal of Marketing. v. 71, p.160-177, 2007.

MCDONAGH, D.; GOGGIN, N.; SQUIER, J. Signs, symbols, and subjectivity: An alternative view of the visual. Computers and Composition. v. 22, p.79-86, 2005.

PETTERSSON, R. Verbo-Visual Communication Verbo-Visual Communication. The 4th International Research Symposium on Visual Verbal Literacy. p. 9, 1996.

PLATTS, K.; HUA TAN, K. Strategy visualisation: knowing, understanding, and formulating. Management Decision, v. 42(5), pp.667-676, 2004.

RIVERA, J.; VIDAL, R.; CHULVI, V.; LLOVERAS, J. La transmisión visual de la información como estímulo cognitivo de los procesos creativos. Anales de Psicologia. v. 26, p.226-237, 2010.

RODRIGUEZ, L.; DIMITROVA, D. V. The levels of visual framing. Journal of Visual Literacy. v. 30, p.48-65, 2011.

SAROOGHI, H.; LIBAERS, D.; BURKEMPER, A. Examining the relationship between creativity and innovation: A meta-analysis of organizational, cultural, and environmental factors. Journal of Business Venturing. v. 30, pp.714-731, 2013.

SAWYER, R. K. Explaining Creativity: The Science of Human Innovation. Oxford, Nova Iorque, 2a ed. 2012.

SMICIKLAS, M. The power of infographics. Indiana: Pearson Education, 2012.

SOK, P.; O'CASS A. Examining the new product innovation-performance relationship: Optimizing the role of individual-level creativity and attention-to-detail. Industrial Marketing Management. 2015. 
STENBERG, R. J. Triangulating love. In: R. J. Sternberg e M. L. Barnes (Eds.). The psychology of love (pp.119-138). Nova Iorque: Yale University, 1988.

TEGARDEN, D.P. Business Information Visualization. Communications of the AIS. v. 1, p.1-37, 1999.

ZHANG, K. Using visual languages in management. Journal of Visual Languages \& Computing. v. 23, p.340-343, 2012. 